\title{
Pulsed NMR Study of Network Formation in the Course of Bulk Polymerization of Methyl Acrylate
}

\author{
Hiroki Kimoto, Atsuko Fukuda, Atsushi Asano, and Takuzo Kurotsu ${ }^{\dagger}$ \\ Department of Applied Chemistry, National Defense Academy, Hashirimizu 1-10-20, Yokosuka, \\ Kanagawa 239-8686, Japan
}

\begin{abstract}
The proton spin-spin relaxation time $\left(T_{2}\right)$ during the bulk polymerization of methyl acrylate was measured as a function of the reaction time at various temperatures. Three kinds of $T_{2}\left(T_{2 \mathrm{~L}}\right.$ (long), $T_{2 \mathrm{~S}}$ (short) and $T_{2 \mathrm{M}}$ (intermediate)) were obtained as the polymerization proceeded. The fraction of $T_{2 \mathrm{~S}}\left(F_{\mathrm{S}}\right)$ increased sigmoidally at a certain reaction time, while that of $T_{2 \mathrm{~L}}\left(F_{\mathrm{L}}\right)$ decreased reciprocally. The former corresponded to the amount of a polymer whose molecular weight was sufficiently high enough to cause a tight entanglement that produced a transient network structure; the latter reflected a decrease in the mixture of the monomer and the low molecular weight of the polymer. $T_{2 \mathrm{M}}$ is considered to arise from a relatively mobile region of the entanglement. The relationship between the fractions of $T_{2 \mathrm{~S}}+T_{2 \mathrm{M}}$ and the polymer yield was found to be linear, which led us to monitor the polymer yield in real time during the polymerization in a nondistractive manner. ${ }^{13} \mathrm{C}$ DD (dipolar decoupling)/MAS (magic angle spinning) NMR spectra were also measured to monitor the polymerization process in terms of the molecular motions between the main chain and the side chain in the formation of a network structure. The ${ }^{13} \mathrm{C}$ DD/MAS NMR spectra show that the side chain motion became restricted as well as the main chain when the "Trommosdorff effect" (gel effect) was observed, and a part of the monomers were trapped in the network structure.
\end{abstract}

(Received September 29, 2004; Accepted January 6, 2005)

\section{Introduction}

Pulsed NMR can be used to elucidate certain aspects of the polymer structure and those properties that are affected by the molecular mobility. The spin-spin relaxation time $\left(T_{2}\right)$, which is primarily determined by the relatively slow motion of the polymer chain in the fluid state, was found to be useful for investigating the polymer structural order, chain entanglement and their presence as permanent cross-links. ${ }^{1-3}$ Short polymer chains in a liquid state or at high temperature in the molten state will give rise to a single exponential $T_{2}$ decay. When the molecular weight exceeds a critical value, the spin-spin relaxation deviates from the single exponential behavior. This is explained by the presence of higher molecular weights of molecular entanglements sufficient in number to provide a three dimensional network. ${ }^{4}$ The entanglement of a polymer chain restricts the chain mobility. Since this long-range chain motion affects the NMR relaxation times, the studying $T_{2}$ can provide a sensitive probe with which to investigate the effect of intermolecular coupling on the mobility.

Most of molecular motion studies, however, have focused on polymers in a static state, such as cross-linked rubbers, ${ }^{5}$ polymer melts with non-disperse molecular weight. ${ }^{3}$ From a molecular motional point of view, bulk polymerization is also one of the most interesting targets for pulsed NMR spectroscopy. The reaction is followed by a liquid-to-solid transition accompanied by a change in the polymer structure, the molecular weight distribution and the density. The molecular motion during the

$\doteqdot$ To whom correspondence should be addressed.

E-mail: kurotu@nda.ac.jp course of the reaction is, therefore, continuously influenced particularly by the propagation process, where the entanglement occurs along with a rapid increase in the degree of polymerization. Under such circumstances, samples consist of a mixture of high-polymer, low-molecular weight polymer, oligomers, and a residual monomer where high molecular weight entangled (network structure) molecules co-exist with low molecular weight "free molecules". The constituents' fractions also continuously change during the reaction. In terms of the molecular motion, studying the network formation by a pulsed NMR method in a kinetic process, such as bulk polymerization, is therefore of interest and worth investigating as well as that by the gravimetric method, ${ }^{6-9}$ because the pulsed NMR method provides such a different perspective on the kinetic process of the reaction in real time.

In the present work, the proton spin-spin relaxation time of poly(methyl acrylate) (PMA) was measured in order to study network formation as a function of the reaction time at various temperatures by the pulsed NMR method. The results were considered in conjunction with that of ${ }^{13} \mathrm{C}$ DD/MAS NMR measurements and the polymer yield.

\section{Experimental}

Pulsed NMR measurements were carried out with a JEOL Mu25 spectrometer operating at a frequency of $25 \mathrm{MHz}$. The proton spin-spin relaxation time $\left(T_{2}\right)$ was obtained from the free induction decay (FID), which follows a $90^{\circ}$ pulse. ${ }^{10}$ The pulse interval time was $10 \mathrm{~s}$, which is sufficiently long to get the spins to be in the thermal-equilibrium state. The dead time of the apparatus was $10 \mu$ s. FID signals were accumulated $8-32$ times to improve 


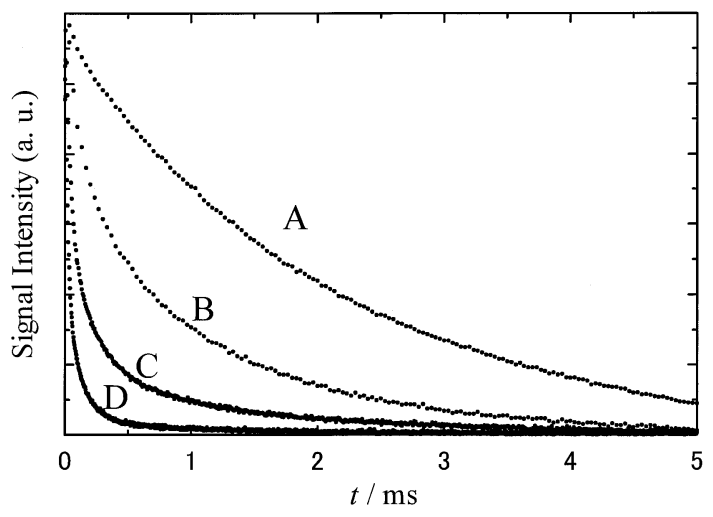

Fig. 1 Typical FID signal change in the polymerization of MA at $55^{\circ} \mathrm{C}$. A, $250 \mathrm{~min} ; \mathrm{B}, 400 \mathrm{~min} ; \mathrm{C}, 500 \mathrm{~min} ; \mathrm{D}, 1800 \mathrm{~min}$.

the signal-to-noise ratio. Methyl acrylate (MA) was distilled under reduced pressure. The monomer was sealed with benzoyl peroxide (BPO) (BPO:MA $=1: 1000$, mole ratio) in a glass tube $(10 \mathrm{~mm} \phi)$ under reduced pressure. Bulk polymerization was carried out in an NMR probe at several temperatures.

${ }^{13} \mathrm{C}$ dipolar decoupling (DD)/magic angle spinning (MAS) NMR spectra were measured by a Bruker DMX 500 spectrometer operating at a carbon frequency of $125 \mathrm{MHz}$. Another experiment concerning the polymerization was also performed under the same experimental conditions as mentioned above to calculate the polymer yield and to carry out ${ }^{13} \mathrm{C}$ DD/MAS NMR measurements. The reaction temperatures were regulated within $\pm 0.5^{\circ} \mathrm{C}$ by an air-flow thermostat. After a certain reaction period, the polymerization was stopped by cooling the sample tube with liquid nitrogen. Poly(methyl acrylate) was precipitated by pouring the reacted methyl acrylate into a large amount of methanol. After the polymer was dried at $80^{\circ} \mathrm{C}$ under reduced pressure, its weight was measured. The polymer yield was calculated by the following equation:

$$
\text { Polymer yield }(\%)=\frac{\text { Weight of produced PMA }}{\text { Weight of MA }} \times 100 \text {. }
$$

\section{Results and Discussion}

Change of free induction decay signal during the course of polymerization

Figure 1 shows a typical change in the FID signals during the course of MA polymerization at $55^{\circ} \mathrm{C}$. The FID signal of MA during the early stage of polymerization was apparently of a single exponential curve with a long spin-spin relaxation time. On the other hand, the decay curve became non-exponential with a short relaxation time after a certain reaction time. It is obvious that the polymerization process reflects the change of the FID signals.

In general, as the polymerization proceeds, methyl acrylate changes from liquid to glassy, or the solid state, via gel or an elastic state. Under such circumstances, the sample at a certain conversion, the $T_{2}$ decays no longer obey a single exponential time dependence.

Thus, the FID curve in a course of polymerization can be fitted to a sum of Weibull functions: ${ }^{4,5}$

$$
S(t)=\Sigma S_{0 \mathrm{i}} \exp \left[-\left(t / T_{2 \mathrm{i}}\right)^{E \mathrm{i}}\right]
$$

Here, $E_{\mathrm{i}}$ is the Weibull coefficient, $S_{0 \mathrm{i}}$ the initial signal intensity of the $\mathrm{i}$-th component at $t=0$, and $T_{2 \mathrm{i}}$ the proton spin-spin

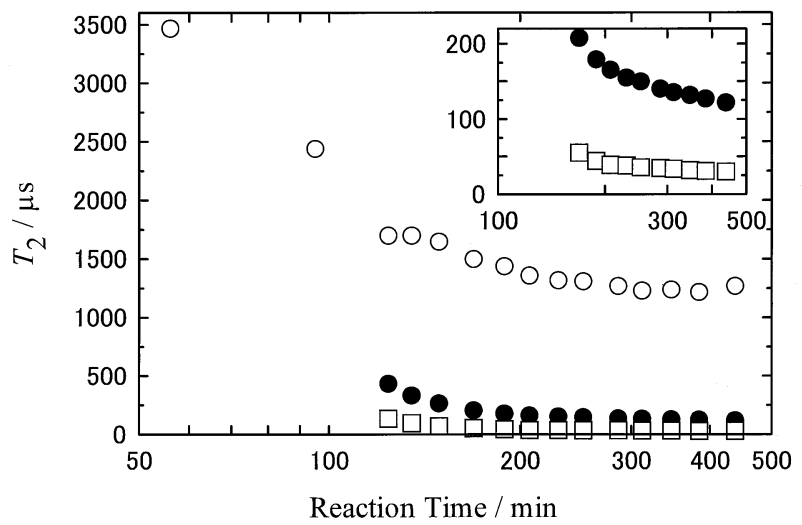

Fig. $2 T_{2 \mathrm{~L}}, T_{2 \mathrm{M}}$, and $T_{2 \mathrm{~S}} v s$. reaction time for $\mathrm{MA}$ at $55^{\circ} \mathrm{C} . \mathrm{O}, T_{2 \mathrm{~L}}$; ○, $T_{2 \mathrm{M}} ; \square, T_{2 \mathrm{~S}}$.

relaxation time of the i-th component. The initial fraction of the $\mathrm{i}$-th component $\left(F_{\mathrm{i}}\right)$ is given by

$$
F_{\mathrm{i}}=S_{0 \mathrm{i}} / \Sigma S_{0 \mathrm{i}}
$$

The Weibull coefficient can be taken as having either a Gaussian $(E=2)$, exponential $(E=1)$ or intermediate character $(1 \leqq E \leqq 2)$. In this experiment, FID signals were found to be exponential at the early stage of polymerization, and became a sum of the exponential and Gaussian components as the polymerization proceeded.

\section{Reaction time dependence of the spin-spin relaxation times}

Figure 2 shows the change in $T_{2}$ of MA as a function of the reaction time at $55^{\circ} \mathrm{C}$. Only a $T_{2}\left(T_{2 \mathrm{~L}}\right)$ was obtained from the analysis of the FID signals at a reaction time less than $100 \mathrm{~min}$. The sample at this stage was still mobile and in the liquid state, containing large amounts of monomers and very low-molecularweight polymers, which allowed a correlation time of less than $10^{-6} \mathrm{~s}$. These constituents of the sample have spectroscopically almost the same motions on a whole. Therefore, the sample yielded only a single exponential component with the Weibull coefficient $(E)$ of 1 . The $T_{2}$ value became smaller than that of the monomer due to an increase in the concentration of the lowmolecular-weight polymer.

At relatively longer reaction times (over $100 \mathrm{~min}$ ), the sample gradually became viscous and then began to solidify. The FID signal with a fast decay part was found to be non-exponential and the part continued to augment with the reaction time. The FID signal at this stage offered three different relaxation times, as analyzed in a manner described by Serizawa et al. ${ }^{4}$ As the polymerization proceeded, monomers decreased, whereas polymers increased. Therefore, it is reasonable to consider that $T_{2 \mathrm{~L}}$ can be assigned to monomers that are mobile and $T_{2 \mathrm{~S}}$ to polymerized molecules that are less mobile. That the longer relaxation time, $T_{2 \mathrm{~L}}(\sim 1.2 \mathrm{~ms})$, remains unchanged, is attributed to mobile species. ${ }^{3,11,12}$ Those are residual monomers and very low-molecular-weight polymers which are trapped among high molecular weight of the polymers during the polymerization. $T_{2 \mathrm{M}}$ also changes from $450 \mu \mathrm{s}$ down to $100 \mu \mathrm{s}$ after this component begins to be observed. Fujimoto et al. pointed out that the low molecular weight of a polymer or a solventimmensed polymer that are relatively mobile species have a longer $T_{2}$ compared to rigid spices $\left(T_{2 \mathrm{~S}} \sim 20 \mu \mathrm{s}\right){ }^{13}$ For an example of a non-network polymer, liner polymers in viscous state such as an epoxy resin, have two relatively long kinds of 
relaxation times of $T_{2}(1 \mathrm{~ms}$ and $300 \mu \mathrm{s}){ }^{14}$ These two kinds of $T_{2}$ are comparable to $T_{2 \mathrm{M}}$ and $T_{2 \mathrm{~L}}$ of this reaction. It is, therefore, expected that the structure of a linear polymer-monomer mixture can be visualized as quasi-entangled mobile molecules.

$T_{2 \mathrm{~S}}$ changed from $c a .100 \mu \mathrm{s}$ to $c a .20 \mu \mathrm{s}$. The shortest relaxation times, $T_{2 \mathrm{~S}}(15-20 \mu \mathrm{s})$, of Gaussian decay is attributed to rigid species that arose from solid aggregation of the high-molecular-weight polymer, amorphous solid. ${ }^{1-3}$ As is often the case with bulk polymerization, the reaction is followed by the "Trommosdorff effect (gel effect)". The polymer chains propagate and begin to interpenetrate each other to form a tight entanglement of polymer chains (transient 3D network structure), which caused the motion of the polymer chain to be constrained..$^{1-3}$ In the previous work, ${ }^{15}$ we also investigated the bulk polymerization process of MMA (methyl methacrylate) by pulsed NMR. The time course of the reaction proceeded in a similar manner to that of MA. The molecular weight of PMMA (poly(methyl methacrylate)) also increased, followed by a "gel effect" to reach a critical value in which entanglements of the polymer chain occurred. Soh et al. pointed out that in a bulk polymerization of MMA, the entanglement of polymer chains occurred as the polymer concentration and/or molecular weight increased. ${ }^{16-19}$ They also explained that the gel effect is closely related to a change of the free volume and the occurrence of the entanglement of a polymer chain whose molecular weight is more than $\mathrm{ca} .10^{4}$.

Information of the molecular weight of PMA in our experiment was not obtained because of the reason mentioned in Ref. 20. The molecular weight of PMA is, however, anticipated to be high enough to cause an occurrence of entanglements of a polymer chain when $T_{2 \mathrm{~S}}$ begins to be observed.

At a higher conversion of the polymerization time, PMA becomes solid, even for a lower-molecular weight polymer $\left(T_{2 \mathrm{~L}}\right)$, and a loosely entangled polymer $\left(T_{2 \mathrm{M}}\right)$ becomes plasticized by a large amount of high-molecular-weight polymers to decrease three kinds of $T_{2}$ values. These values of $T_{2 \mathrm{~S}}, T_{2 \mathrm{M}}$, and $T_{2 \mathrm{~L}}$ are categorically different from each other, and do not merge throughout the whole reaction process. This means that each fraction of different $T_{2}$ belongs to a different mobility region during polymerization.

Reaction time dependence of each fraction of the $T_{2 S}, T_{2 M}$ and $T_{2 L}$ components

Figure 3 shows the change in fraction of each $T_{2 \mathrm{~S}}, T_{2 \mathrm{M}}$, and $T_{2 \mathrm{~L}}$ components, referred to as $F_{\mathrm{S}}, F_{\mathrm{M}}$, and $F_{\mathrm{L}}$, respectively, as a function of the polymerization time at $55^{\circ} \mathrm{C} . F_{\mathrm{S}}$ is zero until the reaction time reaches to $100 \mathrm{~min}$, where it begins to increase sigmoidally to about $50 \%$ at a longer reaction time. On the other hand, $F_{\mathrm{L}}$ decreases abruptly at almost the same time as $F_{\mathrm{S}}$ shows an increase. $F_{\mathrm{M}}$ also appears and then keeps its value at between $c a$. 58 and $40 \%$. In general, as the propagation proceeds, an increase in the amount of the polymer and a decrease in monomer concentration occur. The time course of these fractions, therefore, explains the polymerization process in terms of proton $T_{2}$. The decrease in $F_{\mathrm{L}}$, as mentioned above corresponding to a mixture of monomer and a low-molecularweight polymer, as estimated from its value, was at first observed. At the propagation stage (onset of "gel effect"), those constituents gained molecular weight to transit into a higher molecular-weight region $\left(F_{\mathrm{M}}\right) . \quad F_{\mathrm{M}}$ and $F_{\mathrm{S}}$ were then observed and the amount of $F_{\mathrm{S}}$ increased further with the reaction time through the transition of $F_{\mathrm{M}}$, while keeping its value at between $45-50 \%$. As for all the other polymerizations at different temperatures, the time course of these three kinds of components was similar to that at $55^{\circ} \mathrm{C}$.

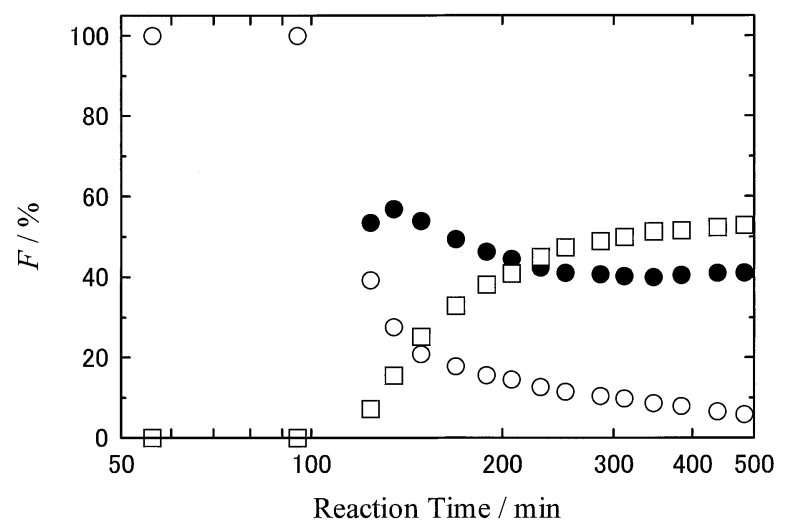

Fig. $3 F_{2 \mathrm{~L}}, F_{2 \mathrm{M}}$, and $F_{2 \mathrm{~S}} v s$. reaction time for $\mathrm{MA}$ at $55^{\circ} \mathrm{C} . \quad \mathrm{O}, F_{2 \mathrm{~L}}$; ○, $F_{2 \mathrm{M}} ; \square, F_{2 \mathrm{~S}}$.

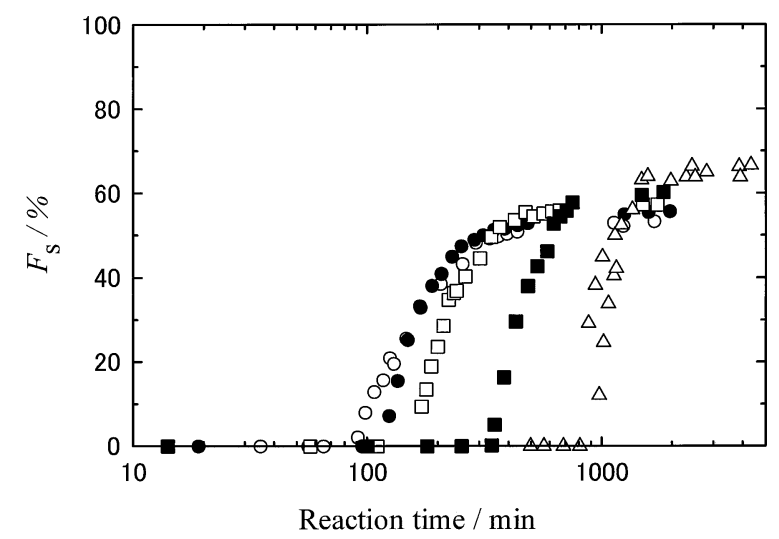

Fig. $4 F_{\mathrm{S}}$ vs. reaction time for $\mathrm{MA}$ at various temperatures. $\mathrm{O}$ $60^{\circ} \mathrm{C} ; \bullet, 55^{\circ} \mathrm{C} ; \square, 50^{\circ} \mathrm{C} ; \mathbf{\square}, 45^{\circ} \mathrm{C} ; \triangle, 40^{\circ} \mathrm{C}$.

\section{Reaction time dependence of $F_{S}$ and the polymer yield}

Figure 4 shows that the dependence of $F_{\mathrm{S}}$ on the polymerization time at various temperatures. $F_{S}$ at each temperature increases sharply at a certain reaction time and reaches a saturated value. ${ }^{15,21}$ This increase occurs at shorter times as the reaction temperature rises. Figure 5 shows the reaction time dependence of the polymer yield at various temperatures. As is well known, in the bulk polymerization of the methacrylate polymer, the yield $v s$. reaction time curve shows a sigmoidal increase due to "gel effect", and has a saturated value. ${ }^{7}$ A similarity in the time course of the reactions can be observed in both Figs. 4 and 5, although there is a difference between the equilibrium values of $F_{\mathrm{S}}$ and the polymer yield. As we have pointed out, $F_{\mathrm{S}}$ corresponds to the amount of a tight entanglement of polymer chains, and $F_{\mathrm{M}}$ corresponds to the amounts of polymers whose molecular weight is lower (more mobile) than that belonging to $F_{\mathrm{S} .}{ }^{15}$ The latter does not form a spectroscopically tight entanglement by itself, or a loosely entanglement. The onset of the gel effect corresponds to the occurrence of entanglement. Taking into account the meaning of $F_{\mathrm{M}}$, we show the time course of $F_{\mathrm{S}}+F_{\mathrm{M}}$ at various temperatures in Fig. 6. The time course of the polymer yield resembles much more that in Fig. 6 than in Fig. 4. Figure 7 shows a comparison of the amount of $F_{\mathrm{S}}+F_{\mathrm{M}}$ and the polymer yield at various temperatures and reaction time. A straight line with a slope of 45 degree was obtained, although the yield slightly exceeded $F_{\mathrm{S}}$ in a region of a polymer yield less 


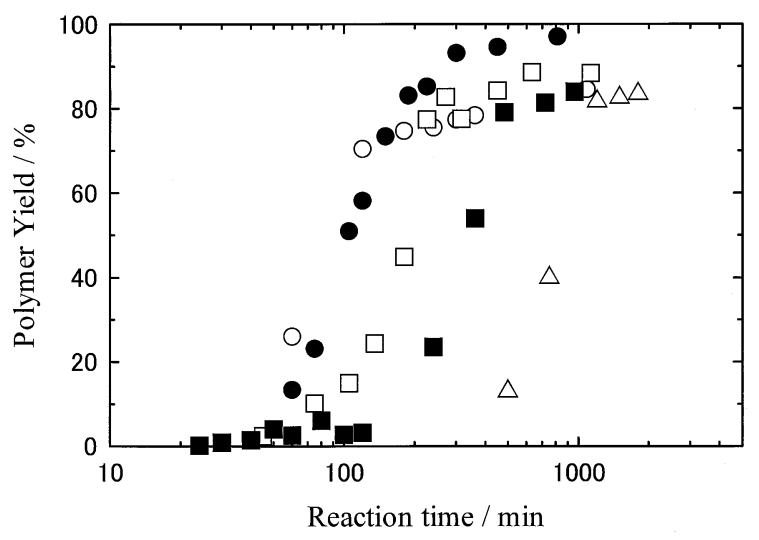

Fig. 5 Polymer yields $v s$. reaction time for $\mathrm{MA}$ at various temperatures. $\mathrm{O}, 60^{\circ} \mathrm{C} ; \bullet, 55^{\circ} \mathrm{C} ; \square, 50^{\circ} \mathrm{C} ; \mathbf{\square}, 45^{\circ} \mathrm{C} ; \triangle, 40^{\circ} \mathrm{C}$.

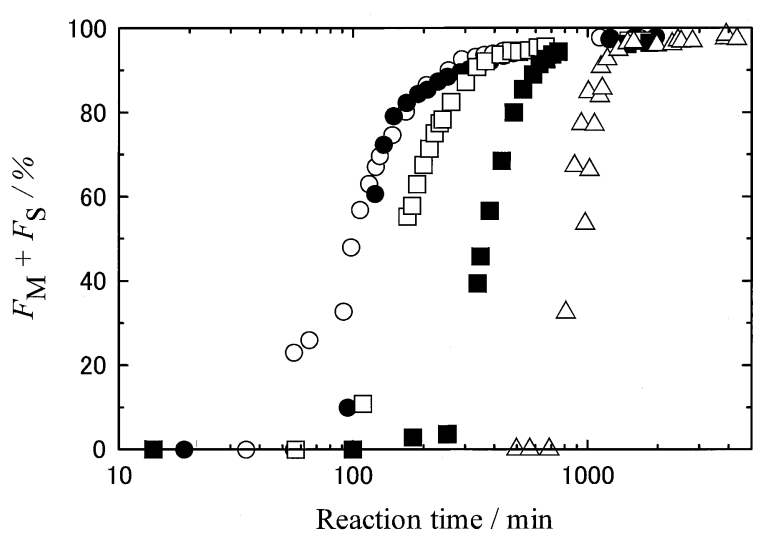

Fig. $6 F_{\mathrm{M}}+F_{\mathrm{S}} v s$. reaction time for $\mathrm{MA}$ at various temperatures. $\mathrm{O}, 60^{\circ} \mathrm{C} ; \bullet, 55^{\circ} \mathrm{C} ; \square, 50^{\circ} \mathrm{C} ; \boldsymbol{\square}, 45^{\circ} \mathrm{C} ; \triangle, 40^{\circ} \mathrm{C}$.

than $20 \%$. At the early stage of polymerization, there was a distribution of the molecular weight of polymers including monomers and oligomers. ${ }^{22}$ Those constituents were observed as the same degree of motion and undistinguishable by this method. Therefore, in this region, we tend to underestimate the values of $F_{\mathrm{S}}+F_{\mathrm{M}}$, compared to the polymer yield.

\section{${ }^{13} \mathrm{C}$ DD/MAS NMR spectroscopy}

${ }^{13} \mathrm{C}$ DD/MAS NMR spectra were also measured on samples recovered after a certain reaction period. Figure 8 shows the ${ }^{13} \mathrm{C}$ DD/MAS NMR spectra of MA or PMA at various reaction times at $55^{\circ} \mathrm{C}$. The carbon peaks belonging to the residual monomers or polymers can be observed separately, which qualitatively offers a spectroscopic view on the cross-section of the polymerization progress. As the polymerization proceeds, the sample becomes viscous and then solidified, and the ${ }^{1} \mathrm{H}-{ }^{13} \mathrm{C}$ dipole-dipole interaction becomes conspicuous, accompanied by a broadening of the peaks. The peaks ascribed from the monomer can still be observed, even at a reaction time of 300 min, and do not disappear. This result supports the idea that a part of $F_{\mathrm{L}}$ is assignable to residual monomers that are trapped, and become less mobile (decrease in $T_{2 \mathrm{~L}}$ ) due to the entangled high-molecular-weight polymers. The peaks of carbons within the main chain become broader with the reaction time as well as those of the carbons of the side chain in polymers, while the width of peak of the monomers remains narrow. These time dependences of the peak broadening correspond to the bulk

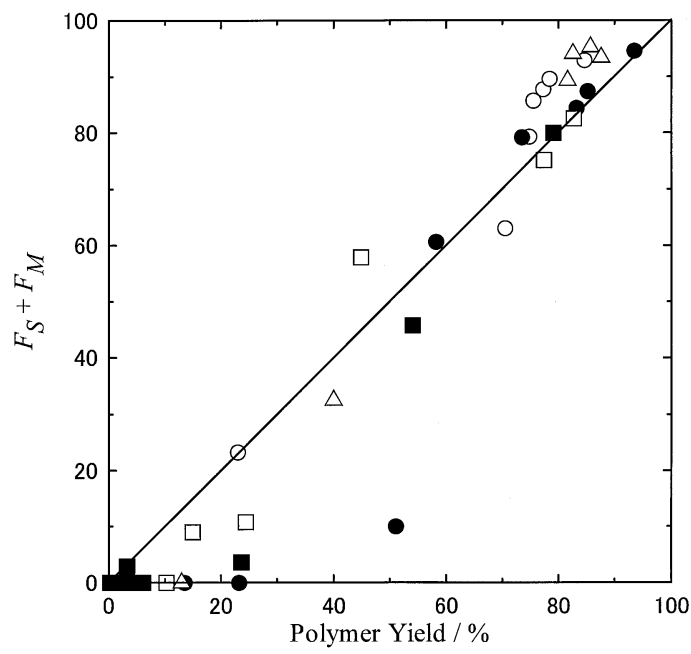

Fig. 7 Relationship between $F_{\mathrm{S}}+F_{\mathrm{M}}$ and polymer yields. $\mathrm{O}, 60^{\circ} \mathrm{C}$; $\bullet, 55^{\circ} \mathrm{C} ; \square, 50^{\circ} \mathrm{C} ; \boldsymbol{\square}, 45^{\circ} \mathrm{C} ; \triangle, 40^{\circ} \mathrm{C}$.
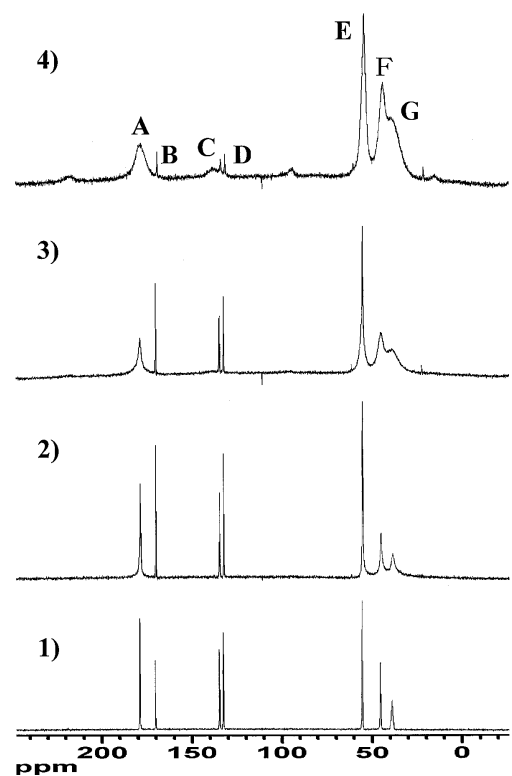

Fig. $8{ }^{13} \mathrm{C}$ DD/MAS NMR spectra for MA and PMA at different reaction times at $55^{\circ} \mathrm{C}$. 1) $30 \mathrm{~min}$, 2) $90 \mathrm{~min}$, 3) $180 \mathrm{~min}$, 4) $300 \mathrm{~min}$. A, $\underline{\mathrm{COOCH}}_{3}$ (polymer); B, $\underline{\mathrm{COOCH}}_{3}$ (monomer); C, $\underline{\mathrm{CH}}_{2}=\mathrm{CH}$; D, $\mathrm{CH}_{2}=\underline{\mathrm{CH}}$; E, $\mathrm{COOCH}_{3}$ (polymer + monomer); F, $-\underline{\mathrm{C}} \mathrm{H}_{2} \mathrm{CH}-; \mathrm{G}$, $-\mathrm{CH}_{2}-\mathrm{C} \mathrm{H}_{-}$.

polymerization process in terms of the mobility of carbon, and also correspond to the results of those of proton $T_{2}$ and their components at $55^{\circ} \mathrm{C}$.

Figure 9 shows an inverse of the half-height full width (FWHH) of the ${ }^{13} \mathrm{C}$ peaks in the main and side chains against the reaction time. The methylene and methine carbons in the main chain become less mobile at a reaction time of $100 \mathrm{~min}$, followed by peak-broadening of the carboxyl carbon, which is near in the position of the main chain. The motion of the methyl carbon at the end of side chain also becomes constrained. Carbons within the main chain become lessmobile at first to form an entanglement, and then affect the motion of the side chain. These results show that the polymerization of MA clearly reflects on the motion of the 


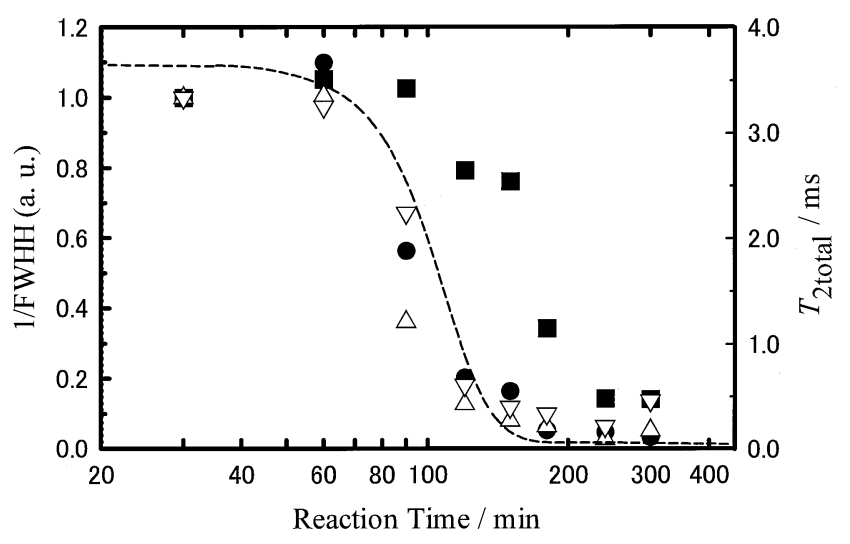

Fig. 9 Full width at half-height (FWHH) of the MA and PMA peaks vs. reaction time at $55^{\circ} \mathrm{C} . \bullet-\mathrm{COOCH}_{3} ; \mathbf{\square}, \mathrm{COOCH}_{3} ; \triangle$, $-{ }_{-} H_{2} \mathrm{CH}-; \nabla,-\mathrm{CH}_{2} \mathrm{CH}-;$ dashed line represents $T_{2 \text { total }}$. The values of FWHH of each MA peak are normalized by that at $30 \mathrm{~min}$.

carbon as well as the proton.

For a comparison of the molecular motion of ${ }^{1} \mathrm{H}$ and ${ }^{13} \mathrm{C}$ during the course of polymerization, we calculated the total relaxation time, $\left(T_{2 \text { total }}\right)$, as follows:

$$
1 / T_{2 \text { total }}=1 / T_{2 \mathrm{~L}} \times F_{\mathrm{L}}+1 / T_{2 \mathrm{M}} \times F_{\mathrm{M}}+1 / T_{2 \mathrm{~S}} \times F_{\mathrm{S}} .
$$

The time course of $T_{2 \text { total }}$ represents comprehensively the occurrence of a tight entanglement of the polymer chain in terms of the proton motion because $T_{2 \text { total }}$ takes into consideration of both the relaxation time and its fractions at the same time. The time course of the motion for the main chain and the side carbon chains inversely corresponds to that of $1 / T_{2 \text { total }}$ for protons at this temperature.

In bulk polymerization, therefore, the sample becomes solidified after the gel effect occurs, due to the occurrence of entanglement of the polymer chains. The solidification of a sample is mainly due to the main chain entanglement, followed by a hindrance of the side chain.

\section{Conclusions}

In this work, we showed that the polymer entanglement occurred at the onset of the gel effect, where the constituents underwent a transition from a liquid to a solid. This transition reflects the mobility of the ${ }^{1} \mathrm{H}$ and ${ }^{13} \mathrm{C}$ spins in the polymer. As for the proton mobility, three kinds of spin-spin relaxation times $\left(T_{2 \mathrm{~S}}, T_{2 \mathrm{M}}, T_{2 \mathrm{~L}}\right)$ were observed, and the fractions $\left(F_{\mathrm{S}}, F_{\mathrm{M}}, F_{\mathrm{L}}\right)$ with these three relaxation times were obtained as the polymerization proceeded. From an NMR spectroscopic point of view, the time course of the three fractions shows a bulk polymerization progress with a decrease in the monomer and an increase in the polymer concentration. The $T_{2 \mathrm{~L}}$ component arises from molecules that are too small to form an entanglement. The $T_{2 \mathrm{~s}}$ component, on the other hand, arose from a motionally restricted region that contained a tight entanglement of the polymer chains. The $T_{2 \mathrm{M}}$ component of which molecular weight was adequately high belonged to the more mobile segment in the entanglement.

From the results concerning the carbon mobility, entanglement occurs due to the propagation of the main chain of a polymer, resulting in a motionless state of the main chain and the side chain. The residual monomers remain in the entanglement, which contributes to a part of the $F_{\mathrm{L}}$ component. During the course of polymerization after an occurrence of the gel effect, the value of $F_{\mathrm{S}}+F_{\mathrm{M}}$ (amounts of entanglement) is equal to the polymer yield. This finding makes it possible to monitor the polymer yield in real time during polymerization and in a non-destructive manner.

\section{References}

1. R. Folland and A. Charlesby, J. Polym. Sci., Polym. Lett. Ed., 1978, 16, 339.

2. R. Folland, J. H. Steven, and A. Charlesby, J. Polym. Sci., Polym. Phys. Ed., 1978, 16, 104.

3. R. Folland and A. Charlesby, Polymer, 1979, 20, 211.

4. H. Serizawa, M. Ito, T. Kanamoto, K. Tanaka, and A. Nomura, Polym. J., 1982, 14, 149.

5. S. Kaufman, J. Polym. Sci., 1972, 9, 829.

6. L. K. J. Tong and W. O. Kenyou, J. Am. Chem. Soc., 1945, 67, 1278.

7. E. Trommsdorff, H. Kohle, and P. Lagally, Macromol. Chem., 1948, 1, 169.

8. F. A. Bovey and G. V. D. Tiers, J. Polym. Sci., 1960, 44, 173.

9. A. B. Moustafa, M. A. Abd El-Ghaffar, and A. S. Badran, J. Polym. Sci., Polym. Chem. Ed., 1981, 19, 179.

10. This method has an advantage of following a swift change of $T_{2}$ like in this kind of polymerization process, although there is a lacking in preciseness of the value arising from an inhomogeneity of magnetic field. In this experiment $T_{2}$ was measured on the same sample continuously under the same conditions throughout the whole polymerization process. Therefore, the inhomogeneity does not need to seriously be taken into account of.

11. I. Kamel and Charlesby, J. Polym. Sci., Polym. Phys. Ed., 1981, 19, 803.

12. H. Tanaka, J. Appl. Polym. Sci., 1983, 28, 1707.

13. K. Fujimoto, T. Nishi, and R. Kado, Polym. J., 1972, 3, 448.

14. H. Kimoto, T. Uemura, and T. Kurotsu, Mem. Nat. Def. Acad., 2003, 43(2), 1.

15. T. Kurotu, Polym. J., 1986, 18, 859.

16. S. K. Soh and D. C. Sundberg, J. Polym. Sci., Polym. Chem. Ed., 1982, 20, 1299.

17. S. K. Soh and D. C. Sundberg, J. Polym. Sci., Polym. Chem. Ed., 1982, 20, 1315.

18. S. K. Soh and D. C. Sundberg, J. Polym. Sci., Polym. Chem. Ed., 1982, 20, 1331.

19. S. K. Soh and D. C. Sundberg, J. Polym. Sci., Polym. Chem. Ed., 1982, 20, 1345.

20. The bulk polymerization of MA is hard to be controlled under a reduced pressure at different temperatures. We, therefore, used a very small amount of initiator (BPO) to be able to monitor the polymerization process, their preventing explosive propagation. The molecular weight measurements for the recovered polymer were essential to obtain an insight into the relationship between the molecular weight and the $T_{2}$ value and its fraction. However, because of a low percentage of BPO to MA, polymers with a high degree of polymerization (DP) did not dissolve into a good solvent, or even polar ones, such as DMSO or DMAc for measurements of the molecular weight. Therefore, information on the molecular weight of PMA was not obtained, except from a very early stage of polymerization.

21. T. Kurotsu, Polym. J., 1987, 19, 285.

22. H. Tanaka and T. Nishi, J. Chem. Phys., 1986, 85, 6197. 